\title{
EKF-based Enhanced Performance Controller Design for Non-linear Stochastic Systems
}

\author{
Yuyang Zhou, Qichun Zhang, Member, IEEE, Hong Wang, Senior Member, IEEE, Ping Zhou, Senior \\ Member, IEEE and Tianyou Chai, Fellow, IEEE
}

\begin{abstract}
In this paper, a novel control algorithm is presented to enhance the performance of the tracking property for a class of non-linear and dynamic stochastic systems subjected to nonGaussian noises. Although the existing standard PI controller can be used to obtain the basic tracking of the systems, the desired tracking performance of the stochastic systems is difficult to achieve due to the random noises. To improve the tracking performance, an enhanced performance loop is constructed using the EKF-based state estimates without changing the existing closed loop with PI controller. Meanwhile, the gain of the enhanced performance loop can be obtained based upon the entropy optimization of the tracking error. In addition, the stability of the closed loop system is analysed in the mean square sense. The simulation results are given to illustrate the effectiveness of the proposed control algorithm.
\end{abstract}

Index Terms-Non-Gaussian stochastic non-linear systems, minimum entropy criterion, extended Kalman filter, tracking performance enhancement

\section{INTRODUCTION}

The performance enhancement of control systems has always been a common topic in control system reasearch [1]. Since the external disturbance widely exists in various industrial processes [2], the tracking performance can be normally characterized by mathematical expectation and variance for Gaussian linear stochastic systems [3]. However, these existing results cannot be applied to the non-linear and non-Gaussian stochastic systems [4]. Furthermore, the distributions of the system variables are still non-Gaussian even if the non-linear stochastic systems are subjected to Gaussian noises. Therefore, different from the traditional control design using mathematical expectation and variance, entropy can be introduced to evaluate the performance of the non-linear and non-Gaussian stochastic systems [5].

In information theory [6], the concept of the entropy is developed to describe the randomness of the stochastic variables.

The work is supported in part by the PNNL Control of Complex Systems Initiative, and by the National Natural Science Foundation of China under Grant 61621004 and Grant 61333007.

Y. Zhou is with the School of Electrical and Electronic Engineering, the University of Manchester, Manchester, M13 9PL UK. (e-mail: annamada@163.com)

Q. Zhang is with the School of Engineering and Sustainable Development, De Montfort University, Leicester, LE1 9BH UK. (e-mail: qichun.zhang@dmu.ac.uk)

H. Wang is with Pacific Northwest National Laboratory, Richland, WA 99352,USA. (e-mail: hong.wang@pnnl.gov)

P. Zhou and T. Y. Chai are with the State Key Laboratory of Synthetical Automation for Process Industries, Northeastern University, Shenyang 110819, China. (Tel: 86-024-83687794; e-mail: zhouping@mail.neu.edu.cn; tychai@mail.neu.edu.cn)
Based on this concept, various performance criteria have been presented to control and optimize the stochastic systems [2], [7]-[10]. Motivated by these works, the performance criterion is presented to charaterise the randomness of the vector-valued tracking error based on Rényi entropy, where it has been shown that the entropy is an extension of the variance [10].

Due to the simple structure, PI controllers are widely used for industrial processes [11]. Moreover, to guarantee the mathematical expectation of the vector-valued tracking error converges to zero, many performance enhancement algorithms have been developed for PI control, where most of them improve the performance of systems via tuning the parameters of the PI controllers [12]-[15]. However, for many industrial processes, PI parameters are fixed and could not be changed freely [16]. Therefore, designing an algorithm without changing the existing PI controller is important for performance enhancement of PI control based systems.

The control objective in this paper is to minimize the entropy of the tracking error while the existing PI controller is fixed. With the similar objective, a variance-based solution for a class of linear Gaussian stochastic systems was proposed in [17]. Based on the discussion above, this variance-based solution is not suitable for non-linear and non-Gaussian stochastic systems. Therefore, this paper presents a novel control algorithm shown in Fig. 1, where the structure of the controller is divided into two parts: the existing fixed PI controller and a compensative controller. It is clear that the existing PI controller could always ensure the basic requirement of tracking property and the compensative controller forms the performance enhancement loop using the system states. Since the states of many systems are unmeasurable, an appropriate state estimation or filtering algorithm should be applied here to estimate the unmeasurable states of systems. Indeed, filtering and state estimation for the non-linear stochastic system have attracted a lot of attention [18]. Except the classic non-linear filtering algorithms like extednded Kalman filter (EKF) [19] and unscented Kalman filter (UKF) [20], [18] has proposed an recursive filtering algorithm for a class of non-linear timevarying systems with missing measurements and quantisation effects, and [21] has developed an recursive state estimation method for an array of discrete time-varying coupled stochastic complex networks with missing measurements. In this paper, the extended Kalman filter (EKF) is employed to estimate the system states for its simple structure.

The main contribution of this paper is to develop a novel control algorithm to enhance the performance of the tracking property for a class of non-linear and non-Gaussian stochas- 
tic systems without changing the existing PI control loop. Compared with PI controller, the compensative controller uses the full state information estimated by EKF instead of the output information only. In addition, the concept of entropy is employed to characterize the randomness of system. The gain of the compensative controller can be adjusted adaptively to minimize the entropy-based performance criterion, which implies that the randomness of the tracking error is attenuated. The convergency of the tracking error under the mean square sense is proved under some assumptions. Finally, the proposed control algorithm is verified by an experiment example which illustrates the effectiveness.

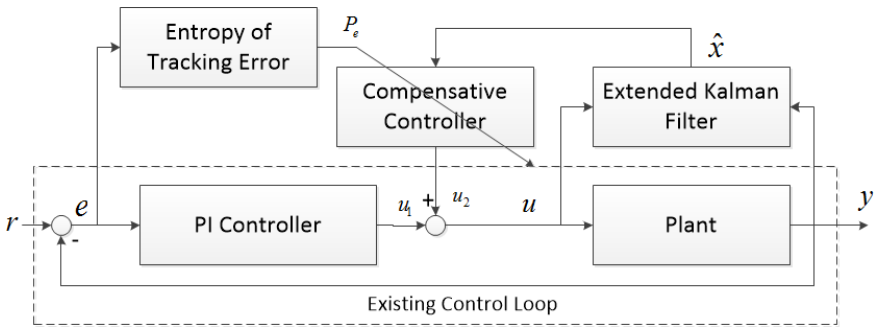

Fig. 1. The block diagram of the proposed control strategy

The rest of this paper is organized as follows: Section II states the formulation of the control problem whilst section III gives the control strategy design which is the main result of this paper. The associated stability analysis is provided in Section IV. Finally, two simulation results and conclusions are given in Section V and Section VI, respectively.

\section{Problem Statement}

Consider the following stochastic non-linear system:

$$
\begin{aligned}
x_{k} & =f\left(x_{k-1}, u_{k-1}\right)+F w_{k} \\
y_{k} & =h\left(x_{k}\right)+Z v_{k}
\end{aligned}
$$

where $x_{k} \in \mathbb{R}^{n}$ is the state vector, $y_{k} \in \mathbb{R}^{m}$ is the system output and $u_{k} \in \mathbb{R}^{m}$ is vector-valued control input. $f(\cdot)$ and $h(\cdot)$ represent general non-linear functions. $w_{k}$ and $v_{k}$ are the process and the measurement noises which are the mutually independent white noises. $Z$ and $F$ are constant coefficient matrices with appropriate dimensions.

The control input could be formulated by Eq.(3) as shown in Fig 1. $u_{1}$ represents the output of the existing PI controller as Eq.(4) while $u_{2}$ is the compensative control signal which takes the full state feedback control (5) based on the estimated states.

$$
\begin{aligned}
u_{k} & =u_{1_{k}}+u_{2_{k}} \\
u_{1_{k}} & =k_{P} e_{k}+k_{I} z_{k} \\
u_{2_{k}} & =G_{k} \hat{x}_{k}^{+}
\end{aligned}
$$

where $k_{P}$ and $k_{I}$ are the fixed parameters in standard PI controller while tracking error vectors $e_{k}$ and $z_{k}$ are described with set-point $r$ as follows.

$$
\begin{aligned}
& e_{k}=r-y_{k} \\
& z_{k}=z_{k-1}+e_{k}
\end{aligned}
$$

In the formula of $u_{2_{k}}, G_{k}$ denotes the compensative gain and $\hat{x}_{k}^{+}$is the posteriori estimation of states from EKF. To obtain the estimated states of the stochastic system, the formulation of EKF is recalled in this section. The prediction step is given as

$$
\begin{gathered}
P_{k}^{-}=A_{k-1} P_{k-1}^{+} A_{k-1}^{T}+F Q_{k-1} F^{T} \\
\hat{x}_{k}^{-}=f_{k-1}\left(\hat{x}_{k-1}^{+}, u_{k-1}\right) \\
A_{k-1}=\left.\frac{\partial f_{k-1}}{\partial x}\right|_{\hat{x}_{k-1}^{+}}, C_{k}=\left.\frac{\partial h_{k}}{\partial x}\right|_{\hat{x}_{k}^{-}}
\end{gathered}
$$

Next, the update stage is shown by Eq.(11)-(13)

$$
\begin{aligned}
K_{k} & =P_{k}^{-} C_{k}^{T}\left(C_{k} P_{k}^{-} C_{k}^{T}+Z R_{k} Z^{T}\right)^{-1} \\
\hat{x}_{k}^{+} & =\hat{x}_{k}^{-}+K_{k}\left(y_{k}-h_{k}\left(\hat{x}_{k}^{-}\right)\right) \\
P_{k}^{+} & =\left(I-K_{k} C_{k}\right) P_{k}^{-}
\end{aligned}
$$

In the above equations, $\hat{x}_{k}^{-}$is the priori state estimation, $\hat{x}_{k}^{+}$represents the posteriori estimated state, $P_{k}^{-}$is the second moment of the priori estimated error and $P_{k}^{+}$is the second moment of the posteriori estimated error. The difference between $\hat{x}_{k}^{-}$and $\hat{x}_{k}^{+}$is that $\hat{x}_{k}^{+}$is much accurate by taking new obtained $y_{k}$ into consideration, which is also the main reason that the posteriori estimated state is chosen to construct the compensative controller. Moreover, $K_{k}$ is the Kalman gain which is obtained by Eq.(11) for each sampling instant. Furthermore, $Q_{k}$ and $R_{k}$ stand for the second moment of the random noises $w_{k}$ and $v_{k}$, respectively.

\section{Control Algorithm Design}

\section{A. Performance Index}

The main purpose is to find an appropriate $G_{k}$ to minimize the randomness of vector-valued tracking error, $e_{k}$. To achieve this control objective, quadratic Rényi's entropy of the vectorvalued tracking error is used and the definition is given by

$$
H_{2}(e)=-\ln (\Omega(e))
$$

where $\Omega(e)$ stands for the information potential and

$$
\Omega(e)=\int \gamma^{2}(e) d e
$$

where $e$ in Eq.(15) stands for the random variable of $e_{k}$ and $\gamma_{e}$ represents the probability density function (PDF) of vectorvalued tracking error. Hence, to calculate the entropy of vectorvalued tracking error, the formula of vector-valued tracking error $e_{k}$ and its PDF $\gamma_{e}$ should be obtained first.

The vector-valued tracking error $e_{k}$ can be restated as follows:

$$
e_{k}=r-y_{k}=H\left(r, x_{k-1}, u_{k-1}, \tau_{1}, v\right)
$$

It is assumed that function $H(\cdot)$ is monotonic with respect to $\tau_{1}$ while $\tau_{1}$ and $v$ denote the random variables of $w_{k}$ and $v_{k}$.

In order to obtain the PDF of the tracking error, the following assumption need to be clarified.

Assumption 1. The Jacobian determinant $\Xi_{k}$ satisfies

$$
\Xi_{k}=\left|\operatorname{det} \frac{\partial H^{-1}\left(r, x_{k-1}, u_{k-1}, \tau_{1}, \tau_{2}\right)}{\partial \tau_{2}}\right| \neq 0
$$


where $\tau_{2}$ stands for the random variable of $e_{k}$ [22]. In addition, two new vectors need to be defined as:

$$
\begin{aligned}
\Phi_{k} & =\left[H^{T}\left(x_{k}, u_{k}, \tau_{1}, v, r\right), w_{k}^{T}\right]^{T} \\
\Psi_{k} & =\left[v_{k}^{T}, w_{k}^{T}\right]^{T}
\end{aligned}
$$

Indeed, Assumption 1 is not a strict condition and it means that in practice the tracking error is always subjected to the non-zero influence of the random disturbances.

Lemma 1. Based upon Assumption 1, the PDF of the tracking error is given by

$$
\begin{aligned}
\gamma_{e_{k}}\left(\tau_{2}\right)=\int_{a}^{b} \gamma_{v}\left(H^{-1}\left(r, x_{k-1}, u_{k-1}, \tau_{1}, \tau_{2}\right)\right) \gamma_{w}\left(\tau_{1}\right) \\
\times\left|\operatorname{det} \frac{\partial H^{-1}\left(r, x_{k-1}, u_{k-1}, \tau_{1}, \tau_{2}\right)}{\partial \tau_{2}}\right| d \tau_{1}
\end{aligned}
$$

where $\gamma_{v}$ and $\gamma_{w}$ are the PDFs of $v_{k}$ and $w_{k}$, respectively, and $H^{-1}\left(r, x_{k-1}, u_{k-1}, \tau_{1}, \tau_{2}\right)$ satisfies the following equation.

$$
\tau_{2}=H\left(r, x_{k-1}, u_{k-1}, \tau_{1}, H^{-1}\left(r, x_{k-1}, u_{k-1}, \tau_{1}, \tau_{2}\right)\right)
$$

Proof. From the probability theory, it can be seen that

$$
\gamma_{\Phi_{k}}\left(\tau_{2}, \tau_{1}\right)=\gamma_{\Psi_{k}}\left(v, \tau_{1}\right)\left|\operatorname{det} \frac{\partial H^{-1}\left(r, x_{k-1}, u_{k-1}, \tau_{1}, \tau_{2}\right)}{\partial \tau_{2}}\right|
$$

where $\gamma_{\Phi_{k}}$ and $\gamma_{\Psi_{k}}$ stand for the joint PDF of $\Phi_{k}$ and $\Psi_{k}$, respectively. Since $w_{k}$ and $v_{k}$ are mutually independent, we have

$$
\gamma_{\Psi_{k}}\left(v, \tau_{1}\right)=\gamma_{v}(v) \gamma_{w}\left(\tau_{1}\right)
$$

Moreover, since

$$
\gamma_{e_{k}}\left(\tau_{2}\right)=\int_{a}^{b} \gamma_{\Phi_{k}}\left(\tau_{2}, \tau_{1}\right) d \tau_{1}
$$

$\gamma_{e_{k}}\left(\tau_{2}\right)$ can be further expressed as

$$
\begin{aligned}
\gamma_{e_{k}}\left(\tau_{2}\right)=\int_{a}^{b} & \gamma_{\Psi_{k}}\left(H^{-1}\left(r, x_{k-1}, u_{k-1}, \tau_{1}, \tau_{2}\right), \tau_{1}\right) \\
& \times\left|\operatorname{det} \frac{\partial H^{-1}\left(r, x_{k-1}, u_{k-1}, \tau_{1}, \tau_{2}\right)}{\partial \tau_{2}}\right| d \tau_{1}
\end{aligned}
$$

Following Lemma 1, the performance index can be formulated using entropy. In addition, the energy of the control inputs should also be considered. Therefore, the performance index function is chosen as follows.

$$
\begin{aligned}
J_{k} & =R_{1} H_{2_{k}}+\frac{1}{2} u_{2_{k}^{T}}^{T} R_{2} u_{2_{k}} \\
& =-R_{1} \ln \int_{\alpha_{1}}^{\beta_{1}} \gamma_{e_{k}}^{2}\left(\tau_{2} \mid x_{k}, \hat{x}_{k}^{+}, G_{k-1}\right) d \tau_{2}+\frac{1}{2} u_{2_{k}}^{T} R_{2} u_{2_{k}}
\end{aligned}
$$

where weights $R_{1}$ and $R_{2}$ are pre-specified real positive numbers.

\section{B. Controller Design}

To minimize $J_{k}$, optimal $G_{k}$ can be solved from

$$
\frac{\partial J_{k}}{\partial G_{k-1}}=0
$$

For each iteration, the parameters are updated in the direction of negative gradient. The optimization procedure is stated as follows.
Initialize $G_{k}$ and set the accuracy value $\bar{\varepsilon}>0$; Calculate the gradient as the following equation

$$
\nabla J_{k}=\left.\frac{\partial J_{k}}{\partial G_{k-1}}\right|_{G_{k}=G_{k-1}}
$$

If $\nabla J_{k} \nabla J_{k}^{T}<\bar{\varepsilon}$, stop the algorithm and the optimal solution is $G_{k-1}$. Otherwise, go to Step 3;

3 The gain is updated using the following equation.

$$
G_{k}=G_{k-1}-\left.\eta \frac{\partial J_{k}}{\partial G_{k-1}}\right|_{G_{k}=G_{k-1}}
$$

where $\eta>0$ is the learning rate;

4 Set $k=k+1$ and go to Step 2.

Remark 1. In order to guarantee the sufficiency of this optimization, the value of $R_{1}$ and $R_{2}$ should be chosen to satisfy the following.

$$
\frac{\partial^{2} J_{k}}{\partial G_{k-1} G_{k-1}^{T}}>0
$$

It has been shown that inequality (30) always holds once the weight $R_{2}$ is sufficiently large.

\section{Stability ANALYSis}

In this section, the conditions of stability in the mean square sense for closed-loop is analysed. For this purpose a new vector $\varepsilon_{k}$ is defined as

$$
\varepsilon_{k}=\left[\begin{array}{lll}
e_{k}^{T} & \eta_{k}^{T} & z_{k}^{T}
\end{array}\right]^{T}
$$

where $\eta_{k}=x_{k}-\hat{x}_{k}^{+}$is the estimated error.

Assumption 2. The original non-linear stochastic system can be approximated by the following formulation

$$
\begin{aligned}
x_{k} & \approx A x_{k-1}+B u_{k-1}+F w_{k} \\
& +\Delta f_{k-1}\left(x_{k-1}, u_{k-1}\right) \\
y_{k} & \approx C x_{k}+Z v_{k}+\Delta h_{k}\left(x_{k}\right)
\end{aligned}
$$

where $\Delta f_{k-1}\left(x_{k-1}, u_{k-1}\right)$ and $\Delta h_{k}\left(x_{k}\right)$ could be considered as non-linear unmodelled dynamics. In the rest of paper, $\Delta f_{k-1}$ and $\Delta h_{k}$ will be applied to represent $\Delta f_{k-1}\left(x_{k-1}, u_{k-1}\right)$ and $\Delta h_{k}\left(x_{k}\right)$ for convenient. The real constant matrices $A, B$ and $C$ can be obtained by linearisation.

Hence, the expression of $e_{k}$ is rewritten as

$$
\begin{aligned}
e_{k} & \approx r-C\left(A x_{k-1}+B u_{k-1}+F w_{k}\right. \\
& \left.+\Delta f_{k-1}\right)-Z v_{k}-\Delta h_{k}
\end{aligned}
$$

Substituting control inputs (3)-(5) to the system, $e_{k}$ can be further expressed as

$$
\begin{aligned}
e_{k} \approx & -C B k_{P} e_{k-1}+C B G_{k-1} \eta_{k-1}-C B k_{I} z_{k-1} \\
& -C F w_{k}-C\left(A+B G_{k-1}\right) x_{k-1}-C \Delta f_{k-1} \\
& -\Delta h_{k}-Z v_{k}+r
\end{aligned}
$$

Similarly, the equation of $\eta_{k}$ can be restated as follows.

$$
\begin{aligned}
\eta_{k} & \approx x_{k}-\hat{x}_{k}^{-} \\
& -K_{k}\left(C x_{k}+Z v_{k}+\Delta h_{k}-C \hat{x}_{k}^{-}-\Delta h_{2 k}\right)
\end{aligned}
$$


where $\Delta h_{2 k}$ stands for the unmodelled dynamics for EKF. Denote

$$
\Delta h_{k}-\Delta h_{2 k}=\xi_{k}\left(x_{k}, \hat{x}_{k}^{-}, v_{k}\right)
$$

then Eq. (36) can be rewritten as

$$
\begin{aligned}
\eta_{k} \approx & \left(I-K_{k} C\right) A \eta_{k-1}+\left(I-K_{k} C\right) F w_{k} \\
& +\left(I-K_{k} C\right)\left(\Delta f_{k-1}-\Delta f_{2 k-1}\right)-K_{k} \xi_{k}-K_{k} Z v_{k}
\end{aligned}
$$

while $z_{k}$ can be formulated by

$$
\begin{aligned}
z_{k}= & z_{k-1}+e_{k} \\
\approx & \left(I-C B k_{I}\right) z_{k-1}-C B k_{P} e_{k-1}+C B G_{k-1} \eta_{k-1} \\
& -C F w_{k}-C\left(A+B G_{k-1}\right) x_{k-1} \\
& -C \Delta f_{k-1}-\Delta h_{k}-Z v_{k}+r
\end{aligned}
$$

and $\varepsilon_{k}$ is re-expressed as

$$
\begin{aligned}
\varepsilon_{k}= & \bar{A}_{k-1} \varepsilon_{k-1}+\bar{F}_{k} w_{k}+\bar{E}_{k} v_{k} \\
& +F_{1_{k}} \Delta f_{k-1}+H_{1} \Delta h_{k} \\
& +A_{2_{k-1}} x_{k-1}+F_{2_{k}} \Delta f_{2 k-1}+H_{2_{k}} \xi_{k}+M r
\end{aligned}
$$

where $\quad \bar{E}_{k}=\left[\begin{array}{lll}-I & -K_{k} & -I\end{array}\right]^{T} Z, \quad F_{1_{k}}=$ $\left[\begin{array}{lll}-C & I-K_{k} C & -C\end{array}\right]^{T}, H_{1}=\left[\begin{array}{ccc}-I & 0 & -I\end{array}\right]^{T}, \bar{F}_{k}=$ $F_{1_{k}} F, A_{2_{k-1}}=H_{1} C\left(A+B G_{k-1}\right), F_{2_{k}}=$ $\left[\begin{array}{ccc}0 & I-K_{k} C & 0\end{array}\right]^{T}, H_{2_{k}}=\left[\begin{array}{lll}0 & K_{k} & 0\end{array}\right]^{T}, M=-H_{1}$ and

$$
\bar{A}_{k-1}=\left[\begin{array}{ccc}
-C B k_{P} & C B G_{k-1} & -C B k_{I} \\
0 & \left(I-K_{k} C\right) A & 0 \\
-C B k_{P} & C B G_{k-1} & \left(I-C B k_{I}\right)
\end{array}\right]
$$

In addition, the following assumptions and lemmas should be used.

Assumption 3. The non-linear unmodelled dynamics of system satisfy the following inequalities:

$$
\begin{aligned}
\Delta f_{k-1} & \leq L_{1}\left\|x_{k-1}\right\|, \Delta h_{k-1} \leq L_{2}\left\|x_{k-1}\right\| \\
\Delta f_{2_{k-1}} & \leq L_{3}\left\|\hat{x}_{k-1}^{+}\right\|, \Delta h_{2_{k-1}} \leq L_{4}\left\|\hat{x}_{k-1}^{-}\right\|
\end{aligned}
$$

where $L_{1}, L_{2}, L_{3}$ and $L_{4}$ are real positive numbers while $\|\cdot\|$ denotes Euclidean norm.

Assumption 4. The following inequality holds with a real positive number $L_{5}$

$$
\left\|\Delta h_{k}-\Delta h_{2_{k}}\right\|=\left\|\xi_{k}\right\| \leq L_{5}\left\|x_{k}-\hat{x}_{k}^{-}\right\|
$$

Noticed that there exists a group of real positive numbers $a_{1}, a_{2}$ and $a_{3}$, such that the following inequalities hold.

$$
\begin{aligned}
\left\|\hat{x}_{k-1}^{+}\right\| \leq a_{1}\left\|x_{k-1}\right\|,\left\|\hat{x}_{k-1}^{-}\right\| & \leq a_{2}\left\|x_{k-1}\right\| \\
\left\|z_{k-1}\right\| & \leq a_{3}\left\|x_{k-1}\right\|
\end{aligned}
$$

Lemma 2. Based on Assumptions 2-4, the following inequality holds.

$$
\left\|\xi_{k}\right\| \leq M_{2}\left\|x_{k-1}\right\|+L_{5}\|F\|\left\|w_{k}\right\|
$$

where $M_{2}=L_{5}\left[\left(1+a_{1}\right) A+L_{1}+a_{1} L_{3}\right]$.
Proof. Based on Assumption 4, inequality (44) can be further expressed as

$$
\begin{aligned}
\left\|\xi_{k}\right\| \leq & L_{5} \| A x_{k-1}+B u_{k-1}+F w_{k}+\Delta f_{k-1}-A \hat{x}_{k-1}^{+} \\
& \quad-B u_{k-1}-\Delta f_{2_{k-1}} \| \\
\leq & L_{5}\|A\|\left\|x_{k-1}-\hat{x}_{k-1}^{+}\right\|+L_{5}\|F\|\left\|w_{k}\right\|+L_{5}\left\|\Delta f_{k-1}\right\| \\
& \quad+L_{5}\left\|\Delta f_{2_{k-1}}\right\|
\end{aligned}
$$

According to Assumption 2 and 3, we have

$$
\begin{aligned}
\left\|\xi_{k}\right\| \leq & \left(1+a_{1}\right) L_{5}\|A\|\left\|x_{k-1}\right\|+L_{5}\|F\|\left\|w_{k}\right\| \\
& +L_{5} L_{1}\left\|x_{k-1}\right\|+a_{1} L_{5} L_{3}\left\|x_{k-1}\right\|
\end{aligned}
$$

The proof is completed by using $M_{2}$.

Lemma 3. Since the above assumptions holds, there exists a real positive number $M_{1}$ such that the following inequality holds.

$$
\begin{aligned}
\Delta h_{k} \leq & M_{1}\left\|x_{k-1}\right\|+\left\|L_{2}\right\|\|F\|\left\|w_{k}\right\| \\
& +\left\|L_{2}\right\|\|B\|\left\|k_{p}\right\|\|r\| \\
& +\left\|L_{2}\right\|\|B\|\left\|k_{p}\right\|\|Z\|\left\|v_{k-1}\right\|
\end{aligned}
$$

where

$$
\begin{aligned}
M_{1}= & \left\|L_{2}\right\|\|A\|+\|C\|\left\|L_{2}\right\|\|B\|\left\|k_{p}\right\| \\
& +\left\|L_{2}\right\|^{2}\|B\|\left\|k_{p}\right\|+a_{3}\left\|L_{2}\right\|\|B\|\left\|k_{i}\right\| \\
& +a_{1}\left\|L_{2}\right\|\|B\|\left\|G_{k-1}\right\|+\left\|L_{2}\right\|\left\|L_{1}\right\|
\end{aligned}
$$

Proof. Based upon Assumption 2, it can be shown that

$$
\begin{aligned}
\Delta h_{k} \leq & \left\|L_{2}\right\|\|A\|\left\|x_{k-1}\right\|+\left\|L_{2}\right\|\|B\|\left\|k_{p}\right\|\left\|e_{k-1}\right\| \\
& +\left\|L_{2}\right\|\|B\|\left\|k_{i}\right\|\left\|z_{k-1}\right\|+\left\|L_{2}\right\|\|B\|\left\|G_{k-1}\right\|\left\|\hat{x}_{k-1}^{+}\right\| \\
& +\left\|L_{2}\right\|\|F\|\left\|w_{k}\right\|+\left\|L_{2}\right\|\left\|\Delta f_{k-1}\right\|
\end{aligned}
$$

Note that $\left\|e_{k-1}\right\|$ can be expressed as

$$
\left\|e_{k-1}\right\| \leq\|r\|+\|C\|\left\|x_{k-1}\right\|+\|Z\|\left\|v_{k-1}\right\|+\left\|\Delta h_{k-1}\right\|
$$

Based on Assumption 3, $\left\|e_{k-1}\right\|$ is represented as

$$
\left\|e_{k-1}\right\| \leq\|r\|+\|C\|\left\|x_{k-1}\right\|+\|Z\|\left\|v_{k-1}\right\|+\left\|L_{2}\right\|\left\|x_{k-1}\right\|
$$

Combining Assumption 2, Assumption 3 and inequality (53), the following inequality can be obtained.

$$
\begin{aligned}
\Delta h_{k} \leq & M_{1}\left\|x_{k-1}\right\|+\left\|L_{2}\right\|\|F\|\left\|w_{k}\right\|+\left\|L_{2}\right\|\|B\|\left\|k_{p}\right\|\|r\| \\
& +\left\|L_{2}\right\|\|B\|\left\|k_{p}\right\|\|Z\|\left\|v_{k-1}\right\|
\end{aligned}
$$

which completes the proof.

Using the above assumptions and lemmas, the following theorem shows that the tracking errors are ultimately bounded in the mean square sense [23], [24], where the definition [25] is shown as below.

Definition 1. For stochastic system in Eq. (1), $x$ is mean square bounded if

$$
E\left(\left\|x_{k}\right\|^{2}\right) \leq+\infty
$$


Theorem 1. Suppose that all the assumptions $A_{1}-A_{4}$ hold, if there exist real positive constants $\delta>0, \rho>0$, such that $\left\|\bar{A}_{k-1}\right\|=\rho<1$ and

$$
\begin{aligned}
& E\left\{\left(\left\|M_{3}\right\|\left\|x_{k-1}\right\|+\left\|T_{1}\right\|\left\|w_{k}\right\|+\left\|T_{2}\right\|\left\|v_{k}\right\|\right.\right. \\
& \left.\left.+\left\|T_{3}\right\|\|r\|+\left\|T_{4}\right\|\left\|v_{k-1}\right\|\right)^{2}\right\} \\
& \leq(1-\rho)^{2} \delta^{2}
\end{aligned}
$$

then for all $E\left\{\left\|\varepsilon_{0}\right\|^{2}\right\} \leq \delta^{2}$, the system is ultimately bounded in the mean square sense, where

$$
\begin{aligned}
M_{3} & =\left\|L_{1}\right\|\left\|F_{1_{k}}\right\|+\left\|M_{1}\right\|\left\|H_{1}\right\| \\
& +\left\|A_{2_{k-1}}\right\|+\left\|L_{2}\right\|\left\|F_{2 k}\right\|+\left\|M_{2}\right\|\left\|H_{2_{k}}\right\| \\
\left\|T_{1}\right\| & =\left\|\bar{F}_{k}\right\|+\left\|H_{1}\right\|\left\|L_{2}\right\|\|F\|+\left\|H_{2_{k}}\right\|\left\|w_{k}\right\| \\
\left\|T_{2}\right\| & =\left\|\bar{E}_{k}\right\| \\
\left\|T_{3}\right\| & =\|M\|+\left\|H_{1}\right\|\left\|L_{2}\right\|\|B\|\left\|k_{p}\right\| \\
\left\|T_{4}\right\| & =\left\|H_{1}\right\|\left\|L_{2}\right\|\|B\|\left\|k_{p}\right\|\|Z\|
\end{aligned}
$$

Proof. Based on Eq.(40), the following equation can be obtained.

$$
\begin{aligned}
\varepsilon_{k}^{T} \varepsilon_{k}= & \varepsilon_{k-1}{ }^{T} \bar{A}_{k-1}^{T} \bar{A}_{k-1} \varepsilon_{k-1}+2 \varepsilon_{k-1}{ }^{T} \bar{A}_{k-1}^{T} \\
& \times\left(\bar{F}_{k} w_{k}+\bar{E}_{k} v_{k}+F_{1_{k}} \Delta f_{k-1}+H_{1} \Delta h_{k}\right. \\
& +A_{2_{k-1}} x_{k-1}+F_{2_{k}} \Delta f_{2_{k-1}}+H_{2_{k}} \xi_{k} \\
& +M r)+\left(\bar{F}_{k} w_{k}+\bar{E}_{k} v_{k}+F_{1_{k}} \Delta f_{k-1}\right. \\
& +H_{1} \Delta h_{k}+A_{2_{k-1}} x_{k-1}+F_{2_{k}} \Delta f_{2_{k-1}} \\
& \left.+H_{2_{k}} \xi_{k}+M r\right)^{T}\left(\bar{F}_{k} w_{k}+\bar{E}_{k} v_{k}\right. \\
& +F_{1_{k}} \Delta f_{k-1}+H_{1} \Delta h_{k}+A_{2_{k-1}} x_{k-1} \\
& \left.+F_{2_{k}} \Delta f_{2_{k-1}}+H_{2_{k}} \xi_{k}+M r\right)
\end{aligned}
$$

As a result, we have

$$
\begin{aligned}
E\left\{\varepsilon_{k}^{T} \varepsilon_{k}\right\}= & E\left\{\varepsilon_{k-1}^{T} \bar{A}_{k-1}^{T} \bar{A}_{k-1} \varepsilon_{k-1}\right\} \\
& +E\left\{2 \varepsilon _ { k - 1 } { } ^ { T } \overline { A } _ { k - 1 } ^ { T } \left(\bar{F}_{k} w_{k}+\bar{E}_{k} v_{k}\right.\right. \\
& +F_{1_{k}} \Delta f_{k-1}+H_{1} \Delta h_{k}+A_{2_{k-1}} x_{k-1} \\
& \left.\left.+F_{2_{k}} \Delta f_{2 k-1}+H_{2_{k}} \xi_{k}+M r\right)\right\} \\
& +E\left\{\left(\bar{F}_{k} w_{k}+\bar{E}_{k} v_{k}+F_{1_{k}} f_{k-1}\right.\right. \\
& +H_{1} \Delta h_{k}+A_{2_{k-1}} x_{k-1}+F_{2_{k}} \Delta f_{2 k-1} \\
& \left.+H_{2_{k}} \xi_{k}+M r\right)^{T}\left(\bar{F}_{k} w_{k}+\bar{E}_{k} v_{k}\right. \\
& +F_{1_{k}} f_{k-1}+H_{1} \Delta h_{k}+A_{2_{k-1}} x_{k-1} \\
& \left.\left.+F_{2_{k}} \Delta f_{2 k-1}+H_{2_{k}} \xi_{k}+M r\right)\right\}
\end{aligned}
$$

Notice that

$$
\begin{aligned}
E\left(\bar{F}_{k} w_{k}\right. & +\bar{E}_{k} v_{k}+F_{1_{k}} \Delta f_{k-1}+H_{1} \Delta h_{k}+A_{2_{k-1}} x_{k-1} \\
& \left.+F_{2_{k}} \Delta f_{2_{k-1}}+H_{2_{k}} \xi_{k}+M r\right) \\
\leq & E\left\{\left\|M_{3}\right\|\left\|x_{k-1}\right\|+\left\|T_{1}\right\|\left\|w_{k}\right\|+\left\|T_{2}\right\|\left\|v_{k}\right\|\right. \\
& \left.+\left\|T_{3}\right\|\|r\|+\left\|T_{4}\right\|\left\|v_{k-1}\right\|\right\}
\end{aligned}
$$

Thus, the following inequality is obtained when $k=0$

$$
\begin{aligned}
E\left\{\varepsilon_{1}^{T} \varepsilon_{1}\right\} \leq & \left\|\bar{A}_{0}\right\|^{2} E\left\{\left\|\varepsilon_{0}\right\|^{2}\right\}+2\left\|\bar{A}_{0}\right\| E\left\{\left\|\varepsilon_{0}\right\|\right\} \\
& \times E\left\{\left\|M_{3}\right\|\left\|x_{0}\right\|+\left\|T_{1}\right\|\left\|w_{1}\right\|+\left\|T_{2}\right\|\left\|v_{1}\right\|\right. \\
& \left.+\left\|T_{3}\right\|\|r\|+\left\|T_{4}\right\|\left\|v_{0}\right\|\right\}+E\left\{\left(\left\|M_{3}\right\|\left\|x_{0}\right\|\right.\right. \\
& +\left\|T_{1}\right\|\left\|w_{1}\right\|+\left\|T_{2}\right\|\left\|v_{1}\right\|+\left\|T_{3}\right\|\|r\| \\
& \left.\left.+\left\|T_{4}\right\|\left\|v_{0}\right\|\right)^{2}\right\} \\
< & \rho^{2} \delta^{2}+2 \rho(1-\rho) \delta^{2}+(1-\rho)^{2} \delta^{2}=\delta^{2}
\end{aligned}
$$

Based on the above inequality, it can be shown that there exists a constant $\theta_{1} \in(0,1)$, such that

$$
E\left\{\varepsilon_{1}^{T} \varepsilon_{1}\right\} \leq \theta_{1}^{2} \delta^{2}
$$

Similarly, $E\left\{\varepsilon_{2}{ }^{T} \varepsilon_{2}\right\}$ can be constructed as follows.

$$
\begin{aligned}
E\left\{\varepsilon_{2}^{T} \varepsilon_{2}\right\} \leq & \left\|\bar{A}_{1}\right\|^{2} E\left\{\left\|\varepsilon_{1}\right\|^{2}\right\}+2\left\|\bar{A}_{1}\right\| E\left\{\left\|\varepsilon_{1}\right\|\right\} \\
& \times E\left\{\left\|M_{3}\right\|\left\|x_{1}\right\|+\left\|T_{1}\right\|\left\|w_{2}\right\|\right. \\
& \left.+\left\|T_{2}\right\|\left\|v_{2}\right\|+\left\|T_{3}\right\|\|r\|+\left\|T_{4}\right\|\left\|v_{1}\right\|\right\} \\
& +E\left\{\left(\left\|M_{3}\right\|\left\|x_{1}\right\|+\left\|T_{1}\right\|\left\|w_{2}\right\|\right.\right. \\
& +\left\|T_{2}\right\|\left\|v_{2}\right\|+\left\|T_{3}\right\|\|r\| \\
& \left.\left.+\left\|T_{4}\right\|\left\|v_{1}\right\|\right)^{2}\right\} \\
< & \rho^{2} \theta_{1}^{2} \delta^{2}+2 \theta_{1} \rho(1-\rho) \\
= & \left(\rho \theta_{1}+(1-\rho)\right)^{2} \delta^{2} \\
\leq & \theta_{2}^{2}\left(\rho \theta_{1}+(1-\rho)\right)^{2} \delta^{2}
\end{aligned}
$$

where $\theta_{2}$ is also a constant with $\theta_{2} \in(0,1)$.

Hence, the following inequality can be constructed at sampling instant $k(k \geq 2)$ by defining $\Theta_{k}{ }^{2}=$ $\left(\sum_{i=1}^{k-1} \tilde{\theta}_{k i}-\rho \sum_{i=1}^{k-2} \tilde{\theta}_{k i}+1-\rho\right)^{2}$ where $\tilde{\theta}_{k i}=\rho^{i} \prod_{n=1}^{i} \theta_{k-n}, 0<$ $\theta_{k}<1, k \geq 2$, such that

$$
E\left(\varepsilon_{k}^{T} \varepsilon_{k}\right) \leq \theta_{k}^{2} \Theta_{k}^{2} \delta^{2}
$$

Therefore, for sampling instant $k+1$, we have

$$
\begin{aligned}
E\left\{\varepsilon_{k+1}^{T} \varepsilon_{k+1}\right\} \leq & \left\|\bar{A}_{k}\right\|^{2} E\left\{\left\|\varepsilon_{k}\right\|^{2}\right\}+2\left\|\bar{A}_{k}\right\| E\left\{\left\|\varepsilon_{k}\right\|\right\} \\
& \times E\left\{\left\|M_{3}\right\|\left\|x_{k}\right\|+\left\|T_{1}\right\|\left\|w_{k+1}\right\|\right. \\
& +\left\|T_{2}\right\|\left\|v_{k+1}\right\|+\left\|T_{3}\right\|\|r\| \\
& \left.+\left\|T_{4}\right\|\left\|v_{k}\right\|\right\}+E\left\{\left(\left\|M_{3}\right\|\left\|x_{k}\right\|\right.\right. \\
& +\left\|T_{1}\right\|\left\|w_{k+1}\right\|+\left\|T_{2}\right\|\left\|v_{k+1}\right\| \\
& \left.\left.+\left\|T_{3}\right\|\|r\|+\left\|T_{4}\right\|\left\|v_{k}\right\|\right)^{2}\right\} \\
< & \rho^{2} \theta_{k}^{2} \Theta_{k}^{2} \delta^{2}+2 \theta_{k} \Theta_{k} \rho(1-\rho) \delta^{2} \\
& +(1-\rho)^{2} \delta^{2} \\
= & \left(\rho \theta_{k} \Theta_{k}+(1-\rho)\right)^{2} \delta^{2} \\
\leq & \theta_{k+1}^{2}\left(\rho \theta_{k} \Theta_{k}+(1-\rho)\right)^{2} \delta^{2} \\
= & \theta_{k+1}^{2} \Theta_{k+1}^{2} \delta^{2}
\end{aligned}
$$

The conclusion can be drawn that the equality holds for $k+1$ if its hold for $k$. The proof is completed.

The procedure of the proposed control algorithm can be summarized as follows:

1 Initialize the system parameters, including the second moment $P_{0}^{+}$of the extended Kalman filter; 
linearise the original non-linear stochastic system to design the EKF for unmeasured variables;

3

4

5

6

8

Obtain the system outputs and estimated states;

Formulate the tracking error and the its PDF;

Calculate the entropy and the performance index value;

6 Obtain the optimal gain $G_{k}$ based on convex optimization (29);

Substitute $G_{k}$ to the performance enhancement loop with existing PI controller (3)-(5);

Move to next sampling instant $k=k+1$ and update the system using step 2 .

\section{Simulations}

\section{A. Simulation for a Twin-level Tank System}

The proposed method has been tested on a twin-level tank system as shown in Fig. 2, which consists of no. 1 and 2 tanks, actuators, a pump, and a valve together with flow-rate and level sensors. The water in the pool is injected into tank 1 with quantity of flow via controlling the pulse width modulating (PWM) ratio $u_{2}(k)$, and then flow from tank 2 to tank 1 with quantity of flow through the intercommunicating valve, while the valve opening $u_{1}(k)$ and the discharge valve of 2 are closed.

Based on [26], when $u_{2}(k)$ is applied to the pump as the control input and with the level of tank 1 as the output, the system could be formulated as:

$$
\begin{aligned}
A_{1} \frac{d x_{1}}{d t} & =k_{3} u_{1}-c_{1}-k_{1} \sqrt{x_{1}}+k_{0} \sqrt{x_{2}-x_{1}}+w_{1} \\
A_{2} \frac{d x_{2}}{d t} & =k_{4} u_{2}-c_{2}-k_{3} u_{1}+c_{1}-k_{2} \sqrt{x_{2}} \\
& -k_{0} \sqrt{x_{2}-x_{1}}+w_{2} \\
y & =x_{1}+v
\end{aligned}
$$

where $x_{1}$ stands for the level of tank 1 and $x_{2}$ is the level of tank 2. $A_{1}$ and $A_{2}$ are the cross-sectional area and $c_{1}, c_{2}$ are constant parameters of the valves and pumps. $k_{0}, k_{1}, k_{2}, k_{3}$ and $k_{4}$ denotes the ratio of the valves. In addition, $w$ and $v$ are the process noise and sensor noise, respectively.

Eq. (66) can be further expressed as follows by taking $u_{1}$ and $k_{2}$ as zero. According to the twin-tank level process in practice, other parameters are identified as: $A_{1}=A_{2}=$ $167.4 \mathrm{~cm}^{2}$ while $c_{1}$ is 0 and $c_{2}$ is $2.88 . k_{0}, k_{1}$, and $k_{4}$ are equal to $0.7,0.25,0.1$, respectively.

To apply the proposed algorithm, the system need to be discretized as follows:

$$
\begin{aligned}
x_{1_{k+1}} & =x_{1_{k}}+\left(A_{1}\right)^{-1} h\left(-c_{1}-k_{1} \sqrt{x_{1_{k}}}+k_{0} \sqrt{x_{2_{k}}-x_{1_{k}}}\right) \\
& +w_{1_{k}} \\
x_{2_{k+1}} & =x_{2_{k}}+\left(A_{1}\right)^{-1} h\left(k_{4} u_{2_{k}}-c_{2}+c_{1}-k_{2} \sqrt{x_{2_{k}}}\right. \\
& \left.-k_{0} \sqrt{x_{2_{k}}-x_{1_{k}}}\right)+w_{2_{k}} \\
y_{k} & =x_{1_{k}}+v_{k}
\end{aligned}
$$

where $h$ is the step length. Noise $w_{k}$ is subjected to Gaussian distribution with zero means and 0.1 variance while $v_{k}$ is nonGaussian noise with the following PDFs.

$$
\begin{aligned}
\gamma_{v_{k}} & =0.6\left[\frac{1}{\sqrt{0.02 \pi}} e^{\frac{-(x-0.2)^{2}}{0.02}}\right] \\
& +0.4\left[\frac{1}{\sqrt{0.02 \pi}} e^{\frac{-(x+0.1)^{2}}{0.02}}\right]
\end{aligned}
$$

The input signal is controlled by the existing closed loop PI controller and the compensative controller. Here the parameters of existing PI controller are $k_{P}=75.5$ and $k_{I}=0.07$.

The compensative signal is added after sampling instant $k=6000$ to show the different performance between the PI controller only and the proposed control. The results are shown in the following figures. Fig.3 indicates the output of system, where it can be seen that the output could track the setpoint properly and the randomness has been attenuated after the compensative signal has been added. This conclusion can also be draw using Fig.4, which shows the 3D PDF of tracking errors, where it illustrates that the shape of PDF becomes taller and sharper after $k=6000$. In addition, Fig.7 shows that the estimated states $x_{2}$ as compared with the real state. Moreover, Fig. 5 shows the curves of the compensative gain optimization while Fig. 6 indicates the compensative signal $u_{2}$.

From these figures, it can be seen that the randomness of output has been reduced after the compensative signal is added. It can also be proven by the shape of tracking errors PDF, which becomes taller and sharper along with the optimal gains searching. Compared with the PI control only, the proposed control algorithm can realize a better tracking performance.

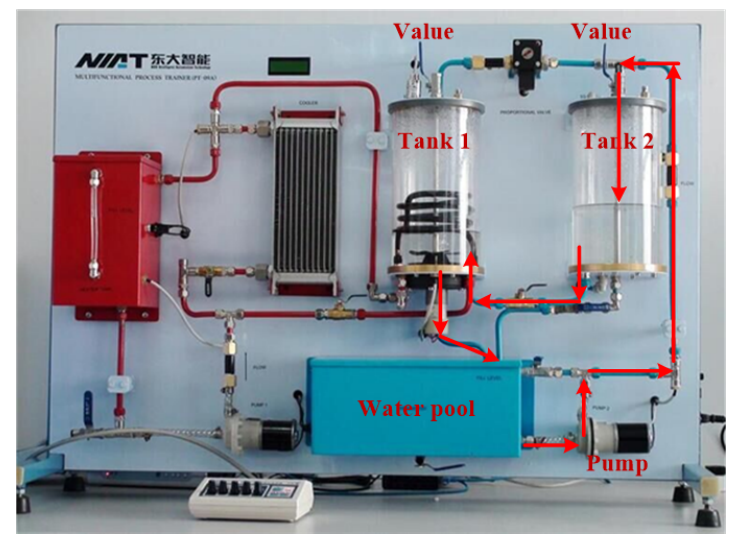

Fig. 2. The structure of the twin-tank level system.

\section{B. Comparison Experiment}

A comparison experiment with minimum variance control is also included. The system formulations and all the parameters are the same as those in the experiment in part A.

The structure of control input can be formed as Eq.(3). Different from the proposed algorithm, the compensative controller is obtained using the minimum variance via the following gradient descent algorithm:

$$
u_{2_{k}}=u_{2_{k-1}}-\left.\eta \frac{\partial J_{k}}{\partial u_{2_{k}}}\right|_{u_{2_{k}}=u_{2_{k-1}}}
$$




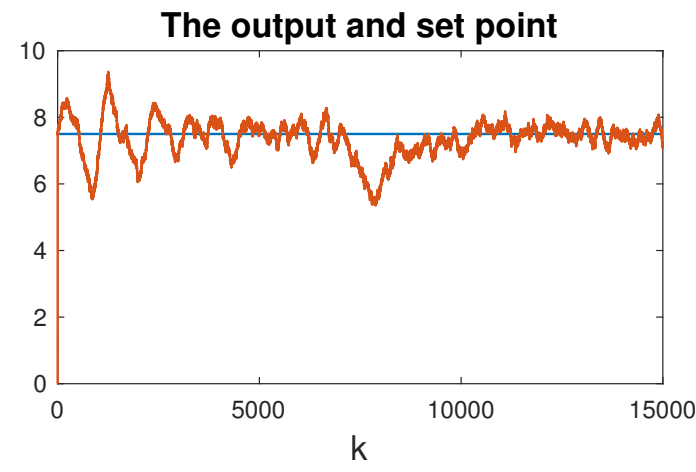

Fig. 3. The response of the closed loop system output.

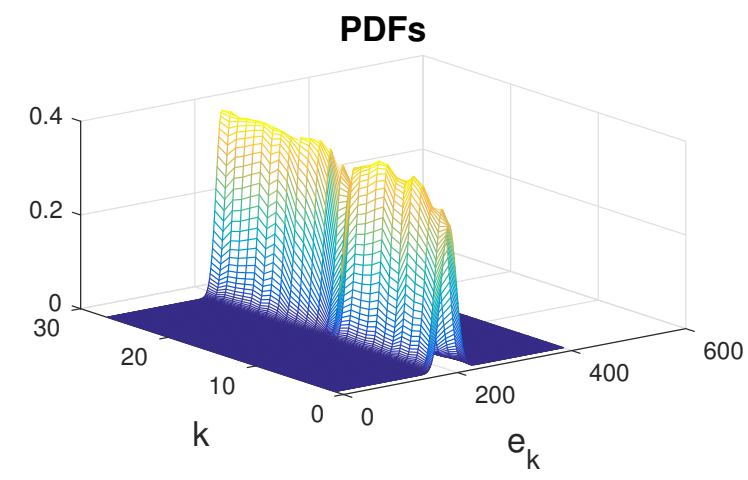

Fig. 4. The 3D response of the PDFs for the tracking errors.

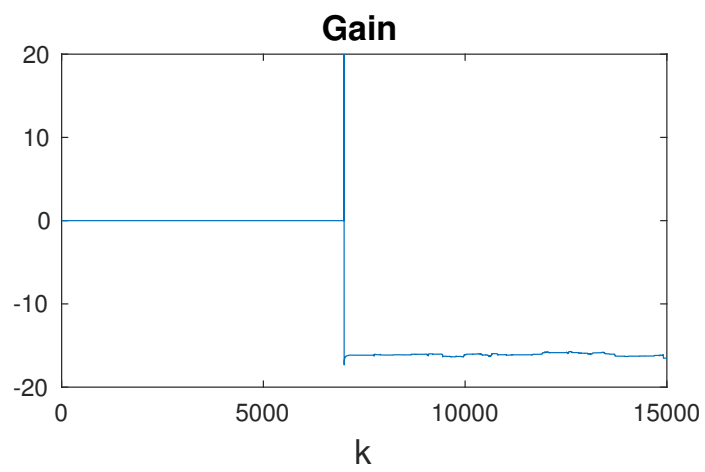

Fig. 5. The responses of the tuned gain for the performance enhancement loop.

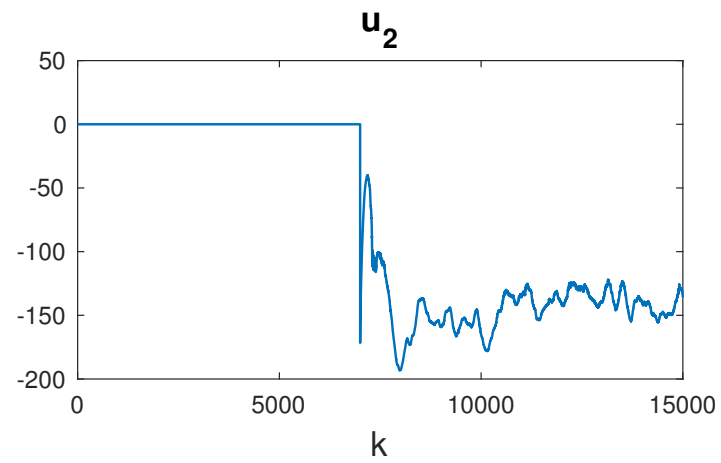

Fig. 6. The response of the compensative signal $u_{2}$.

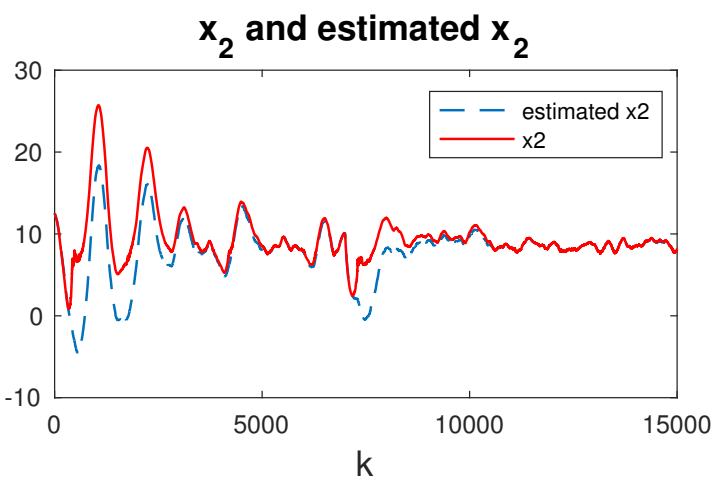

Fig. 7. The responses of state $x_{2}$ and its estimate.

Since the purpose of the designed controller is to minimize the variance of the tracking error, the objective is

$$
J_{k}=R_{1} V_{k}+\frac{1}{2} u_{2_{k}}{ }^{T} R_{2} u_{2_{k}}
$$

where $V_{k}$ is the variance of the tracking error which takes the following form

$$
V_{k}=\int_{a}^{b} x^{2} \gamma_{e_{k}}(x) d x
$$

where $\gamma_{e_{k}}$ is the PDF of the tracking error which can be obtained using Lemma 1.

The results are shown in Fig.8-Fig.10. Similar to the entropy-based experiment, the proposed controller is switched on when time instant $k=6000$ to show the effectiveness. Fig.8 indicates the responses of the system output and set point. The PDFs of the tracking errors are shown in Fig.9 while the compensative signal is illustrated in Fig.10. From Fig.8, it can be seen that the randomness is decreased slightly after the compensative signal is added. Meanwhile the shape of PDFs shown in Fig.9 becomes slightly taller along with the tuning of the gain. Compared with the results of the proposed control algorithm, it is clear that the proposed algorithm shows a better performance.

\section{The output and set point}

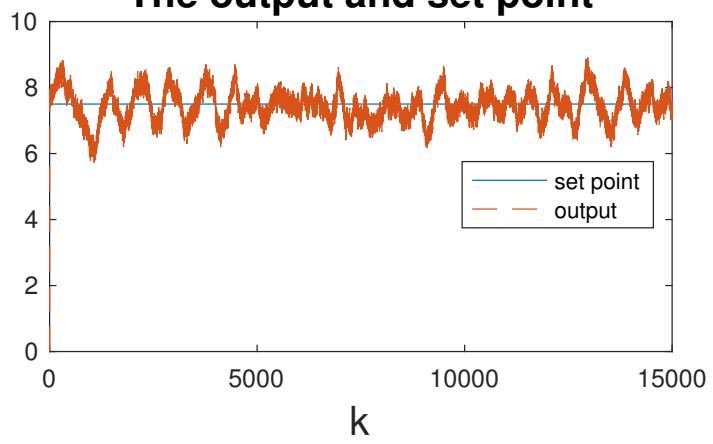

Fig. 8. The response of the closed loop system output.

\section{CONCLUSION}

This paper presents a novel control algorithm for a class of non-linear and non-Gaussian stochastic systems with unmeasurable states. To enhance the tracking performance of 


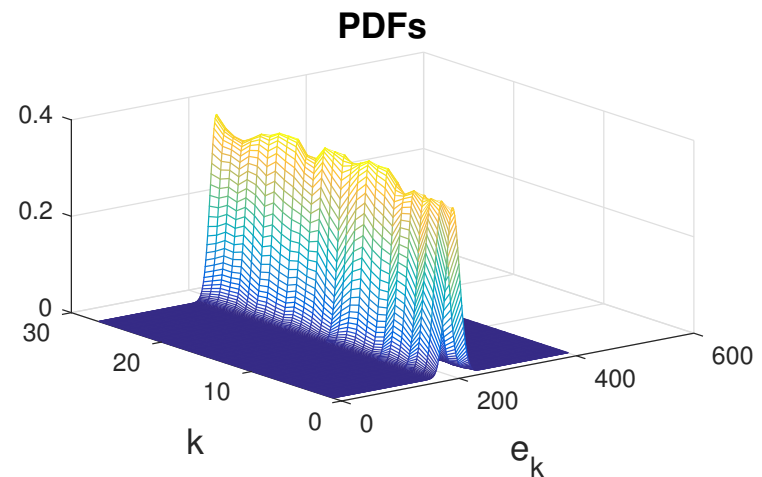

Fig. 9. The 3D response of the PDFs for the tracking errors.

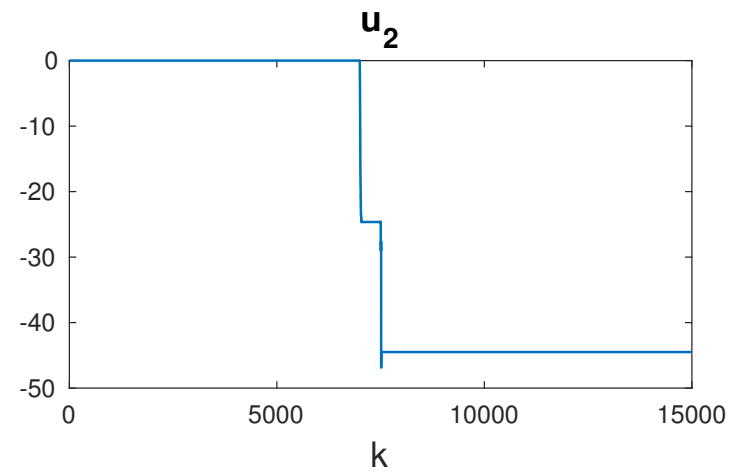

Fig. 10. The response of the compensative signal $u_{2}$

the system, the entropy-based performance index has been developed. Using this performance criteria, the optimal gain has been obtained. Then the performance enhancement loop has been established by this compensative gain and the EKFbased estimated states. Without changing the existing PI control loop, the new control algorithm with compensative control signal attenuate the randomness of the closed-loop system. Moreover, the presented control algorithm is convergent and the stability of the closed-loop stochastic system has been also analysed in the mean square sense under some simple assumptions. To verify this control algorithm, the simulation results have been obtained via one experimental example and one comparison example, and the desired control performances have been obtained.

\section{REFERENCES}

[1] R. M. Murray, K. J. Astrom, S. P. Boyd, R. W. Brockett, and G. Stein, "Future directions in control in an information-rich world," IEEE Control Systems Magazine, vol. 23, no. 2, pp. 20-33, 2003.

[2] M. Ren, J. Zhang, and H. Wang, "Minimized tracking error randomness control for nonlinear multivariate and non-gaussian systems using the generalized density evolution equation," IEEE Transactions on Automatic Control, vol. 59, no. 9, pp. 2486-2490, 2014.

[3] M. J. Grimble, "Controller performance benchmarking and tuning using generalised minimum variance control," Automatica, vol. 38, no. 12, pp. $2111-2119,2002$

[4] X. Liu and Z. Gao, "Robust finite-time fault estimation for stochastic nonlinear systems with brownian motions," Journal of the Franklin Institute, 2016.
[5] H. Wang, "Control of conditional output probability density functions for general nonlinear and non-gaussian dynamic stochastic systems," in Control Theory and Applications, IEE Proceedings-, vol. 150, no. 1. IET, 2003, pp. 55-60.

[6] T. M. Cover and J. A. Thomas, Elements of information theory. John Wiley \& Sons, 2012.

[7] J. Jia, T. Liu, H. Yue, and H. Wang, "Minimum entropy control algorithm for general dynamic stochastic systems," in Innovative Computing, Information and Control, 2006. ICICIC'06. First International Conference on, vol. 1. IEEE, 2006, pp. 368-372.

[8] J. Zhang, M. Ren, and H. Wang, "Minimum entropy control for nonlinear and non-gaussian two-input and two-output dynamic stochastic systems," Control Theory \& Applications, IET, vol. 6, no. 15, pp. 2434$2441,2012$.

[9] L. Guo and H. Wang, "Minimum entropy filtering for multivariate stochastic systems with non-gaussian noises," IEEE Transactions on Automatic Control,, vol. 51, no. 4, pp. 695-700, 2006.

[10] H. Yue and H. Wang, "Minimum entropy control of closed-loop tracking errors for dynamic stochastic systems," IEEE Transactions on Automatic Control, vol. 48, no. 1, pp. 118-122, 2003.

[11] K. J. Åström and T. Hägglund, Advanced PID control. ISA-The Instrumentation, Systems and Automation Society, 2006.

[12] R. K. Mudi and C. Dey, "Performance improvement of pi controllers through dynamic set-point weighting," ISA transactions, vol. 50, no. 2 , pp. 220-230, 2011.

[13] W. Li, X. Chang, J. Farrell, and F. M. Wahl, "Design of an enhanced hybrid fuzzy p+ id controller for a mechanical manipulator," Systems, Man, and Cybernetics, Part B: Cybernetics, IEEE Transactions on, vol. 31, no. 6, pp. 938-945, 2001.

[14] J. Normey-Rico, C. Bordons, and E. Camacho, "Improving the robustness of dead-time compensating pi controllers," Control Engineering Practice, vol. 5, no. 6, pp. 801-810, 1997.

[15] K. Hwu and Y. Yau, "Performance enhancement of boost converter based on pid controller plus linear-to-nonlinear translator," IEEE Transactions on Power Electronics, vol. 25, no. 5, pp. 1351-1361, 2010

[16] T. Chai, S. J. Qin, and H. Wang, "Optimal operational control for complex industrial processes," Annual Reviews in Control, vol. 38, no. 1, pp. 81-92, 2014.

[17] Y. Zhou, Q. Zhang, and H. Wang, "Enhanced performance controller design for stochastic systems by adding extra state estimation onto the existing closed loop control," in 11th UKACC International Conference on Control, 2016. UKACC'11., vol. 1. IEEE, 2016, pp. 368-372.

[18] J. Hu, Z. Wang, B. Shen, and H. Gao, "Quantised recursive filtering for a class of nonlinear systems with multiplicative noises and missing measurements," International Journal of Control, vol. 86, no. 4, pp. $650-663,2013$.

[19] R. E. Kalman and R. S. Bucy, "New results in linear filtering and prediction theory," Journal of Basic Engineering, vol. 83, no. 1, pp. 95-108, 1961.

[20] E. A. Wan and R. Van Der Merwe, "The unscented kalman filter for nonlinear estimation," in Adaptive Systems for Signal Processing, Communications, and Control Symposium 2000. AS-SPCC. The IEEE 2000. Ieee, 2000, pp. 153-158.

[21] J. Hu, Z. Wang, S. Liu, and H. Gao, "A variance-constrained approach to recursive state estimation for time-varying complex networks with missing measurements," Automatica, vol. 64, pp. 155-162, 2016.

[22] L. Yin and L. Guo, "Joint stochastic distribution tracking control for multivariate descriptor systems with non-gaussian variables," International Journal of Systems Science, vol. 43, no. 1, pp. 192-200, 2012.

[23] J. Zhou, D. Zhou, H. Wang, L. Guo, and T. Chai, "Distribution function tracking filter design using hybrid characteristic functions," Automatica, vol. 46, no. 1 , pp. 101-109, 2010

[24] Y. Liu, H. Wang, and L. Guo, "Observer-based feedback controller design for a class of stochastic systems with non-gaussian variables," IEEE Transactions on Automatic Control, vol. 60, no. 5, pp. 1445-1450, 2015.

[25] Z. Gao and X. Shi, "Observer-based controller design for stochastic descriptor systems with brownian motions," Automatica, vol. 49, no. 7, pp. 2229-2235, 2013.

[26] T. Chai, Y. Zhang, H. Wang, C.-Y. Su, and J. Sun, "Data-based virtual unmodeled dynamics driven multivariable nonlinear adaptive switching control," IEEE Transactions on Neural Networks, vol. 22, no. 12, pp. 2154-2172, 2011. 